\title{
The LHC Main Dipoles and Quadrupoles Toward Series Production
}

\author{
Lucio Rossi
}

\begin{abstract}
The Large Hadron Collider (LHC) is under construction at CERN. Most of its $27 \mathrm{~km}$ underground tunnel will be filled with superconducting magnets, mainly $15 \mathrm{~m}$ long dipoles and 3.3 $\mathrm{m}$ long quadrupoles. In total 1248 dipole and 400 quadrupole magnets will be built (including spares), all wound with copper stabilized NbTi Rutherford cables and designed to operate in superfluid helium at $1.9 \mathrm{~K}$. The dipoles operative field is $8.3 \mathrm{~T}$, the system being designed for possible operation up to $9 \mathrm{~T}$ (ultimate field). The pre-series dipole production has started in 2000 in three companies while the series dipole construction will start at the beginning of 2003. As far as quadrupoles are concerned, the prototypal phase (carried out at CEA-Saclay, F) is followed directly by the series construction, started in March 2002. In the paper the magnet main characteristics are reviewed and the special tooling, put in place to fulfill the mass production with the suitable accuracy and safety margin, is described. The results obtained on the first fifteen pre-series magnets as well as the effect of the corrective actions taken to meet the necessary field quality are discussed. Finally a realistic plan of the Project, foreseeing that last dipoles are delivered in summer 2006, is presented.
\end{abstract}

Index Terms-Accelerators, cryogenics, magnets, superconductors.

\section{INTRODUCTION}

$\mathbf{T}$ HE LHC [1]-[3] is a circular accelerator designed to accelerate two counteracting beam protons from injection energy of $0.45 \mathrm{TeV}$ up to a flat top energy of $7 \mathrm{TeV}$, at which the ring is switched from synchrotron mode to collision mode. The main dipole magnets, also called Main Bends (MB's), fill $2 / 3$ of the ring, the remaining tunnel length being dedicated to beam focusing (Main Quadrupole magnets, MQ), to other beam optics functions (chromaticity control, dispersion suppression, etc.) and to beam collision preparation in the Interaction Regions. For the LHC, the $27 \mathrm{~km}$ long underground tunnel, that formerly hosted the LEP machine, is filled with about $18 \mathrm{~km}$ of main dipoles and $1.2 \mathrm{~km}$ of main quadrupoles. The average radius of curvature in the arc is about $2.6 \mathrm{~km}$ and the choice of $8.33 \mathrm{~T}$ as flat top field in the dipoles has determined the collision energy of $7 \mathrm{TeV} /$ beam. As the dipoles are an element of the optic lattice, they must be set to the same field level, or better to the same bending strength, BL, to within a few $10^{-4}$. This means, that poor performance of a dipole cannot be compensated by better performance of another one, thus the weakest dipole will eventually determine the energy performance of the whole machine.

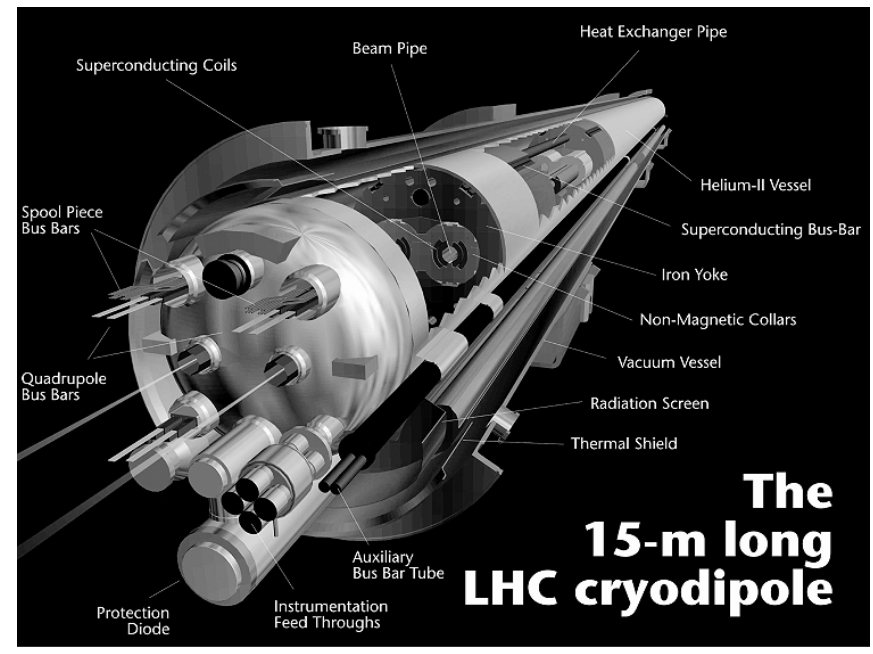

Fig. 1. An artistic view of the LHC dipole in its cryostat.

In total 1232 main dipoles (1104 in the lattice and 128 in the Dispersion Suppression-DS-sections) and 392 main quadrupoles (360 in the lattice and 32 in the DS) will be installed in the tunnel.

Finally, it should be underlined that a very early decision in the Project was to have Two-in-One magnets [4], see Fig. 1, where two channels with opposite field direction for each of the counteracting beams are placed inside the same cold yoke. However, two different schemes were devised for MQ and MB [5]. The former has the coils of the two apertures mechanically independent and magnetically decoupled, while the latter has the two apertures mechanically coupled, through common collars. The absence of iron in between the two apertures of the MB makes the apertures themselves magnetically coupled, too. This concept of strong coupling is summarized with the name of Twin Dipoles. This last solution has some disadvantages in the design and the assembly with respect to the plain Two-in-One scheme but it has the important advantage of reducing the magnet size (an important feature for dipoles) and it has the benefit of a lower assembly cost: the evaluation carried out at the beginning of the Project estimated the cost reduction of the Twin concept, with respect to the separated dipoles concept (like the SSC) of the order of 20\% [6], while the cost advantage of Twin over a simple Two-In-One design may be of the order of $5 \%$.

\section{DiPOLES DESIGN}

\section{A. Main Dipole Evolution}

The design of the LHC MB's has gone through about 10 years of evolution with three generations of design [7]. All based on
The author is with CERN, European Organization for Nuclear Researches, LHC Division, Geneva 23, CH-1211 Switzerland (e-mail: lucio.rossi@cern.ch) Digital Object Identifier 10.1109/TASC.2003.812639 
$\mathrm{NbTi} / \mathrm{Cu}$ Rutherford type cables, all foreseen to work in superfluid helium (a fundamental choice done as early as 1989 [8] to allow to go beyond $8 \mathrm{~T}$ with a sufficient margin) and all featuring two coil layers wound with different cables. The three generations differ in the coil layout, in the collar design and on how the coil-collar assembly interferes with the yoke-skin assembly. The basic design characteristics of the present third-final-generation are:

1) Coil Layout: Based on six-conductor blocks [9]. After an unsuccessful attempt to work with five coil blocks of the second generation, the present design is based on an optimized six block layout, where the conductors are as radial as possible and the shear forces among conductors are minimized. The six blocks leave more room for minor optimizations, i.e., cross-section fine-tuning during series construction, especially in the inner layer that features four blocks.

2) Coil Layers: The two shells are wound with two cables whose margins in critical current are very similar. This feature has improved central field per unit current but implies that imperfections in winding of the second layer are as important as those of the inner layer, despite the considerable number of turns grouped in the two blocks of the outer shell.

3) Collars:: Of the twin type, there are made of special austenitic steel with very low magnetization under operating conditions [10]. They are obtained by fine blanking according to a shape that ensures the wanted coil cavity under stress and cold conditions, and for this reason the collars are slightly elliptical $(\varepsilon=0.1 \mathrm{~mm})$ when punched. The choice of stainless steel, introduced relatively late in the Project after a long period when an aluminum alloy was preferred, [11] is not strictly necessary to reach the design field but allows a more comfortable margin in the construction and assembly tolerances, as shown in [12]. This partly compensates for the higher cost of austenitic steel with respect to aluminum alloy. Also, thanks to its higher rigidity, the use of austenitic steel helps to limit conductor movements, an important issue in magnets where field accuracy is required at $1 \mathrm{~cm}$ from the conductor.

4) Iron: The presence of an iron insert at the vertical symmetry plane in between collar and iron yoke, see Fig. 2, helps the assembly accuracy and the transmission of vertical force from the shell to the collars, through the iron yoke, in a position that is critical for the twin design. Indeed the lack of left-to-right symmetry in twin collars is one of the main disadvantages with respect to the single collaring coil assembly. The inclined surface of the iron insert is meant just to compensate the reduced rigidity in the central arm of each aperture of the collars. The interference between iron yoke and collars is also situated at the mid-plane, the outer arm of each aperture, and at two different positions along the collar outer arm, see Fig. 2, [11], [12]. The iron insert proved to be useful to fine tune the field quality for even harmonics [13].

\section{B. Superconductors}

The NbTi superconducting alloy is characterized by an extremely high homogeneity: $1.0 \%$. The most significant challenges in the production of the 1200 tons of superconducting cable necessary for the LHC Project are [14]:

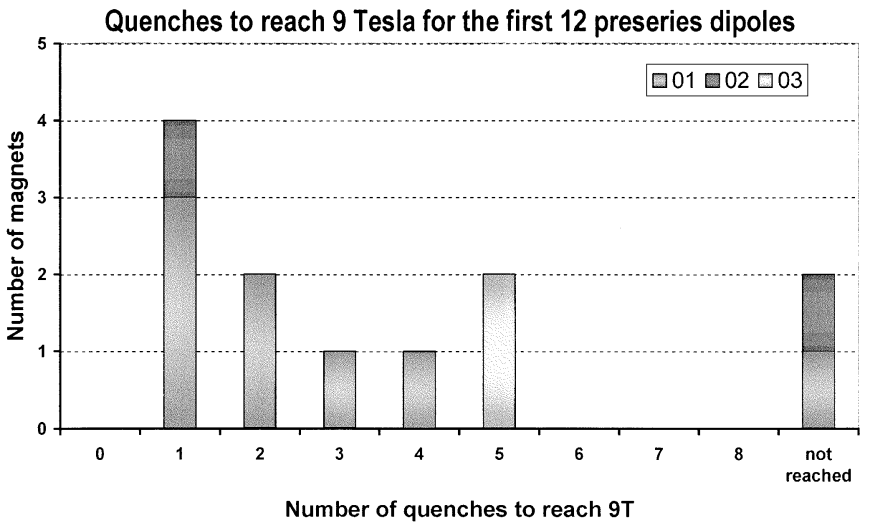

Fig. 2. Cross section of the dipole (only one quadrant) showing the position of interferences between yoke, pushed by shrinking cylinder, and collars. 340 tonnes is the total radial force (including the lower quadrant).

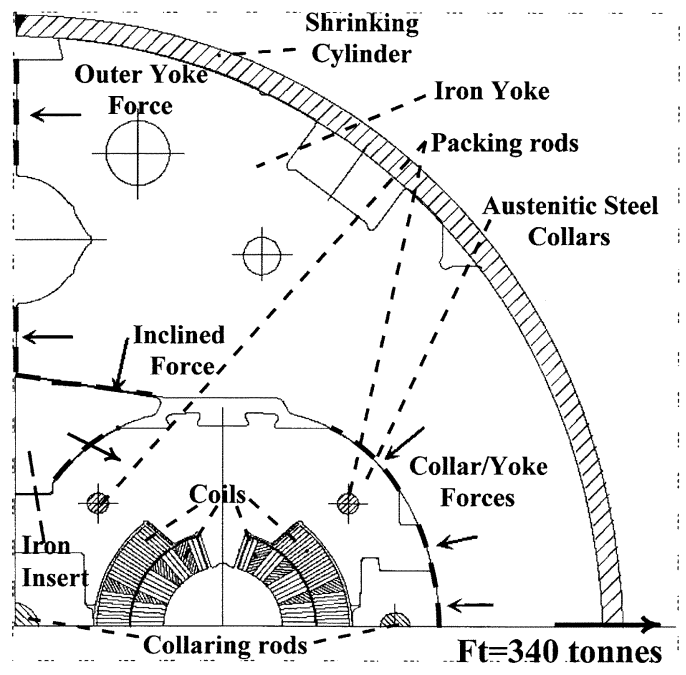

Fig. 3. Interstrand resistance, $R_{c}$, for an inner cable of one contract (last 18 months production). The $20-40 \mu \Omega$ is really at hand (acceptable $15-150$ ).

- A very tough tolerance on the cable sizes, $\pm 6 \mu \mathrm{m}$, requiring both a very strict control of the single wire diameter (usually in the $1 \mu \mathrm{m}$ range!), good compactness (90\%) no sharp edge without brushing.

- Severe limits on magnetization values, to keep under control the persistent currents that can harm the field quality at injection. In fact, magnetization proved to be the parameter most sensitive to the type of manufacturing process but also to changes, variations and nonuniformities in each manufacturing process.

- The control of interstrand resistance. The novel solution adopted for the LHC is based on coating the strands with a $\mathrm{SnAg}$ alloy, before cabling. After cabling, the cable roll is then exposed to a controlled heat treatment in air in order to oxidize the cables. This method is simple, cheap and suitable to Rutherford cabling techniques but required many adjustments and practical $R \& D$ to become reliable in an industrial environment but today is certainly one of the keys to the success of the LHC cables, see Fig. 3.

- The global quality demands, i.e., strict statistical process control limits, no cold welds, no cross-overs, no sharp edges 
TABLE I

CABLES CHARACTERISTICS

\begin{tabular}{lll}
\hline Nominal operating field & $\mathrm{T}$ & 8.33 \\
Ultimate operating field & $\mathrm{T}$ & 9 \\
Quench field from short sample & $\mathrm{T}$ & 9.65 \\
Coil aperture & $\mathrm{mm}$ & 56 \\
Coil thickness (two layers) & $\mathrm{mm}$ & 30.5 \\
Magnetic Length @1.9 K & $\mathrm{m}$ & 14.3 \\
Nominal operating current & $\mathrm{A}$ & 11850 \\
Operating temperature & $\mathrm{K}$ & 1.9 \\
Distance between aperture axes @1.9 K & $\mathrm{mm}$ & 194 \\
Cold Mass Outer diameter & $\mathrm{mm}$ & 570 \\
Overall length of cold mass & $\mathrm{m}$ & 15.18 \\
Mass of the magnet cold mass & $\mathrm{t}$ & 27.5 \\
Stored energy @ 8.3 T, both channels & $\mathrm{MJ}$ & 7.1 \\
Self Inductance (7.6 mH/meter) & $\mathrm{mH}$ & 100 \\
$\Sigma F_{x}$ per unit length per quadrant & $\mathrm{MN} / \mathrm{m}$ & 1.7 \\
Axial e.m. force & $\mathrm{MN}$ & 0.37 \\
\hline \hline
\end{tabular}

(cable brushing is allowed only for the initial production phase), $0.1 \mu \mathrm{m}$ tin coating uniformity, no anomalies, tough limits on the copper content for protection reasons (despite the fact that margins on Jc would allow for a broader range of copper content), complete traceability of all cables, all wires and their individual components. Coupled with the necessity of a good yield, forced by a low price, these conditions require a strict follow up and a powerful dynamically allocated database to exchange information and approval between CERN and industries.

In Table I the main characteristics of the LHC main superconducting cables are reported.

\section{Superconducting Coils}

The coils are composed of poles of two layers each. The necessity to avoid sorting that would certainly slow the production, or make it more complicated, requires that each pole, and even each layer, be identical within $100 \mu \mathrm{m}$ : indeed a $100 \mu \mathrm{m}$ variation in the azimuthal coil size corresponds to a variation of about $0.1 \%$ of the main field, 3.5 and -0.4 units $\left(10^{-4}\right)$ of the main harmonics, sextupole and decapole respectively, and to about 12 $\mathrm{MPa}$ in azimuthal coil pre-stress. A coil with nominal size and compressive modulus (some $12 \mathrm{MPa}$ at room temperature and $17 \mathrm{MPa}$ at cold) will be submitted to $75 \mathrm{MPa}$ pre-stress. Since the allowed range for coil pre-stress is $60-90 \mathrm{MPa}$, if the coils differ more than $125 \mu \mathrm{m}$ from the target they will require a shim thickness different than nominal size, to the detriment of the field quality. This reason together with necessity of top-bottom and left-right symmetry means that coils must all be similar, within the quoted figures.

The main factors to reach accuracy are a good and reproducible winding, a good and accurate curing mould (coil size almost perfectly reproduces the mould geometry, when the mould is closed) and a reliable method and equipment for measurement of the coil size.

Tolerances of components play a fundamental role in achieving long-term accuracy, since the strategy is to avoid single coil shim adaptation for reasons of time and cost. In effect, the components of the coils, most of them procured by CERN, are well inside the requested range, as shown in Fig. 3 for the collars. It should be noted that the variation
TABLE II

MAIN DIPOLES CHARACTERISTICS

\begin{tabular}{lll}
\hline STRAND & Type 01 & Type 02 \\
\hline Diameter $(\mathrm{mm})$ & 1.065 & 0.825 \\
$\mathrm{Cu} / \mathrm{NbT}$ ratio & $1.6-1.7 \pm 0.03$ & $1.9-2.0 \pm 0.03$ \\
Filament diameter $(\mu \mathrm{m})$ & 7 & 6 \\
Number of filaments & 8800 & 6425 \\
$\mathrm{Ic}(\mathrm{A}) @ 1.9 \mathrm{~K}$ & $515( \pm 4 \%) @ 10 \mathrm{~T}$ & $380( \pm 4 \%) @ 7 \mathrm{~T}$ \\
$\mathrm{Jc}\left(\mathrm{A} / \mathrm{mm}^{2}\right) @ 1.9 \mathrm{~K}$ & $1530 @ 10 \mathrm{~T}$ & $2100 @ 7 \mathrm{~T}$ \\
$\mu_{0} \mathrm{M}(\mathrm{mT}) @ 1.9 \mathrm{~K}, 0.5 \mathrm{~T}$ & $30 \pm 4.5$ & $23 \pm 4.5$ \\
\hline CABLE & Type 01 & Type 02 \\
Number of strands & 28 & 36 \\
Width $(\mathrm{mm})$ & 15.1 & 15.1 \\
Mid-thickness $(\mathrm{mm}) @ \mathrm{MPa}$ & $1.900 \pm 0.006$ & $1.480 \pm 0.006$ \\
Keystone angle (degrees) & $1.25 \pm 0.05$ & $0.90 \pm 0.05$ \\
Cable Ic $(\mathrm{A}) @ 1.9 \mathrm{~K}$ & $13750 @ 10 \mathrm{~T}$ & $12960 @ 7 \mathrm{~T}$ \\
Maximum Ic cabling degradation & $5 \%$ & $5 \%$ \\
Interstrand resistance $(\mu \Omega)$ & $10-50$ & $20-80$ \\
\hline
\end{tabular}

of components inside a single coil is necessarily much more stringent than the acceptable variation among lots.

\section{Cold Mass Assembly}

As previously stated, the coil-collar assembly is surrounded by the magnetic circuit contained by a shrinking cylinder, formed by welding two half-shells made out of 316 LN stainless steel. This provides the necessary rigidity to the whole magnet. The forces are transmitted by an interference among very rigid pieces (collars and yoke). Therefore not only the precision of the single pieces is high (typically $\pm 20 \mu \mathrm{m}$ for collars and $\pm 50 \mu \mathrm{m}$ for yoke) but the assembly must ensure this precision as well over the $15 \mathrm{~m}$ magnet length.

It is of some interest to note that near the magnet ends, for $370 \mathrm{~mm}$, the laminations are composed of an outer shell of low carbon steel, like the main body, and with an inner shell, $20 \mathrm{~mm}$ thick of special stainless steel, non magnetic and whose mechanical properties, namely the thermal contraction, have been selected in order to fit exactly that of the iron. These nested laminations [15], are designed to lower the peak field on the coil end (always a quench risk region) while preserving the maximum magnetic length and improving the bad quadrupole coming from aperture coupling at the end.

The magnet must be curved, with a sagitta of about $9 \mathrm{~mm}$, corresponding to a radius of curvature of $2812.36 \mathrm{~m}$. This curvature has a tolerance of $\pm 1 \mathrm{~mm}$, with the exception of the extremities of the magnet where the tolerance is very tight: \pm 0.3 (systematic) and $0.5 \mathrm{~mm}$ r.m.s. in order to keep the corrector magnets centered with respect to the beam tube, to avoid harmonic feed down (detrimental to beam optics).

\section{MAIN QUADRUPOLES}

The design of the MQ's features two apertures completely independent, according to the plain Two-in-One concept.

\section{A. Coil Layout}

In order to save money and complexity over the whole project, the coil is not graded, and the same cable, identical to the one used for the outer layer of the MB's, is employed to wind both layers of the quadrupoles. This means that the magnetic design is not fully optimized but allows the use of 
real double pancake techniques, avoiding the splice between layers in the high field region, a source of concern for the MB's. ${ }^{1}$ Nominal gradient is $223 \mathrm{~T} / \mathrm{m}$, and $241 \mathrm{~T} / \mathrm{m}$ at ultimate machine performance ( $9 \mathrm{~T}$ dipole field) when the peak field in the coils reaches 7.5 T. It is worth notice that although the peak field is somehow reduced in the MQ's with respect to the MB's, the field quality is not. Due to a decision to eliminate the dodecapole (first allowed harmonic in quadrupoles) corrector magnets in the machine, the field quality of all quadrupoles must be excellent. This requires:

- No systematic deviation from the nominal conductor position. Indeed due to the fact that only one longitudinal spacer is present per layer, correction during production is more difficult.

— Repeatability of cable position within $40 \mu \mathrm{m}$.

\section{B. Mechanical Structure}

Each coil aperture is independently collared in a four-fold symmetric vertical press, with strong nonmagnetic austenitic steel single collars, $27 \mathrm{~mm}$ thick. The two apertures are then assembled in a laminated yoke with a central iron arm that decouples the two apertures. Each coil-collar assembly is not supported by the yoke (self supporting collars), that is coupled to the coils only by $3.5 \mathrm{~m}$ long centering and antitorsion keys. As it is well known for quadrupoles the alignment is a critical issue: this is obtained by fitting with dowels the yoke into a very precise (better than $100 \mu \mathrm{m}$ straightness) $5.3 \mathrm{~m}$ long inertia tube. The same inertia tube is used also to ensure the proper alignment for other magnets, like Sextupoles, Octupoles and corrector magnets, forming the so called staright sections. Keeping the tolerances on this assembly will be one of the major challenges in the MQ construction.

A detailed review of the MQ design as well as of the results on the prototyping phase is reported in [16], [17]. In Fig. 4 the cross section of the MQ is reported.

\section{Production Strategy}

Since the early days of the project a few companies were selected as capable to carry out the construction of the MB's. These companies built models and then prototypes of all generations, and finally three were retained for a $3 \times 30$ dipole contract (pre-series) placed in autumn 1999. Then the same companies, the French consortium Alstom MSA-Jeumont Industries, Ansaldo Superconduttori (Italy) and Babcok Noell Nuclear (Germany), were assigned the final contract (series) for further $3 \times 386$ dipoles in spring 2002. The total value of the contracts assigned to these companies exceeds M€ 300.

For the MQ's the strategy was different. Given the size, fulllength prototyping was developed in the laboratory by CEASaclay in collaboration with CERN. Then all European magnet manufacturers were called to assist in the main operations and to tender directly for the total quantity. In 1999 Accel (Germany)

\footnotetext{
${ }^{1}$ So far only one splice failed, thus limiting a $10 \mathrm{~m}$ long dipole model below nominal field. However a pre-series dipole is also suspected to have a resistance of the splice at the limit of the acceptability, having a different behavior at 1.9 $\mathrm{K}$ and at $4.2 \mathrm{~K}$, probably due to different cooling conditions.
}

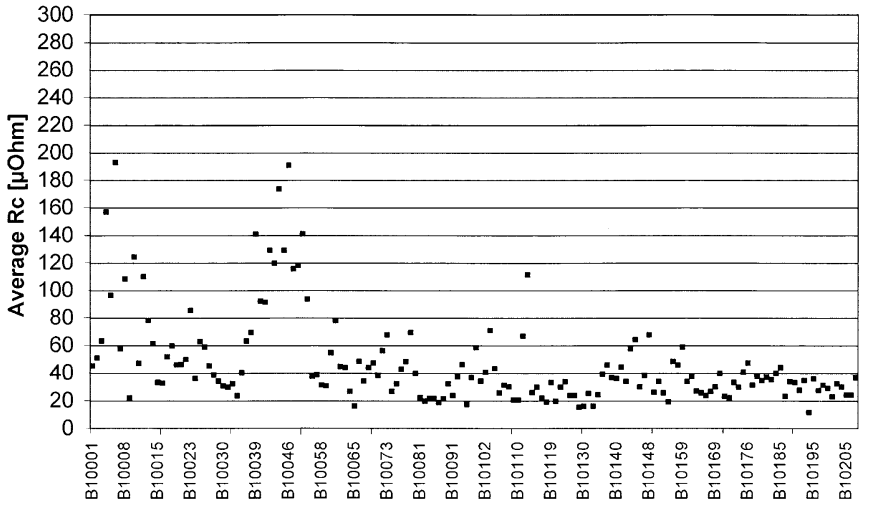

Fig. 4. Cross section of a LHC Main Quadrupole in its cryostat.

was then granted the $M € 35$ contract for the manufacture of all 400 MQ's.

\section{A. CERN Supplied Components}

As part of a cost saving strategy and in order to keep under control the characteristics of the magnet components having some impact on the final quality and on the schedule, all main components are supplied by CERN to the magnet manufacturers. For the main dipoles CERN procures directly:

- Superconducting cables, in single unit lengths, sorted for similar properties for each magnet.

- Polyimide insulation ribbons, both bare and adhesive, as well all polyimide foils for all insulation.

- Longitudinal copper wedges and fine blanked austenitic collars at final shapes.

- End spacers, layer jump boxes, interlayer insulation grooved foils and quench heaters (pre-series only).

- All types of yoke laminations (iron and nested).

- Insulated beam tubes, HeII heat exchanger tubes and 316 LN semi-shells forming the skin, as well as others $15 \mathrm{~m}$ long tubes and the interconnection bellows.

- End covers that, welded to the skin at the two extremities, close longitudinally the cold mass.

- $15 \mathrm{~m}$ long $13 \mathrm{kA}$ bus bars and protection Diodes and all Corrector Magnets of the Dipole Cold Mass.

This strategy implies that CERN becomes supplier of its suppliers with an intricate share of responsibility that makes CERN fully responsible for the magnet performance, except in case of negligence or clear fault of the dipole manufacturer.

The logistics are organized in such a way that only two months storage is kept at the company (one month for cables) and everything is delivered on time according to needs and to technical optimization. All is (or will be, in series production) done via software, through a specifically built database, and warns when components are needed with sufficient lead-time.

A particular case is the cable database. CERN is, for $85 \%$ of the production, supplier of the raw material to the wire and cable manufacturer, requiring to keep under control the production quality and traceability, with approval of each billet and strand map for cabling. Here a sophisticated data base, dynamically allocated and shared among CERN and wire manufacturer, is in use to help to relate all measurements to lots of material and to help on-line approval by CERN. 


\section{B. Large Tooling}

After sound design and good component quality, the next ingredient for high quality and fast production is appropriate tooling.

The tooling related to coil winding and assembly is the responsibility of magnet manufacturers and, although designed according to CERN specifications, is slightly different from company to company. Since very early in the project the companies were involved to modeling and prototyping, and construction of the coils was considered an area where CERN was in charge of monitoring, rather than defining the process.

In contrast, the large presses used for coil collaring and for cold mass welding were specified and procured directly by CERN. Three collaring presses, with a $700 \mathrm{~mm}$ wide and 450 $\mathrm{mm}$ high frame, were designed to have sufficient compressive force, more than $20 \mathrm{MN}$ per meter length, to assure that the collaring was achieved in one step (in addition to the necessary massaging cycles to accommodate conductor) while preserving an extreme accuracy of the $15 \mathrm{~m}$ long beam. The beam alignment and its rigidity assure positioning during operation of about $20 \mu \mathrm{m}$.

Much more complicated has been the design of the welding press. Here many functions have to be assured:

- Delivery of a compressive force, to align laminations and to stress the skin, up to $12 \mathrm{MN}$ per meter length, in a $2000 \mathrm{~mm}$ wide and $1500 \mathrm{~mm}$ high frame.

- Alignment of the $15 \mathrm{~m}$ long cold mass according to the a shape that, once the shells are welded, ensures that at rest the magnet has the right curvature.

- Automatic synchronous (both sides) welding equipment, capable to carry out the welds of the $10 \mathrm{~mm}$ thick 316 LN half-shells in a single working shift. The weld must guarantee good quality and a regular pre-stress of about $150 \pm 30 \mathrm{MPa}$ and, of course, be leak tight to about $10^{-11}$ mbar $1 \mathrm{~s}^{-1}$.

Together with the press manufacturer, ${ }^{2}$ and after considerable $\mathrm{R} \& \mathrm{D}$, it was chosen to have the root pass welded by STT (speed: $70 \mathrm{~mm} /$ minute) and then to fill the seam with three passes of MIG (200 mm/min.). STT is a rather new process, used in industry for only a few years and not yet industrialized for automatic welding on austenitic steel. Its development and practical implementation took some time and presents some drawbacks (for example it is very sensitive to change of filler wire) but it has some great advantages:

1) Through a sophisticated electronics and parameter control at milliseconds level, is able to cope with different conditions, although the most important parameters, the welding speed, cannot be adjusted independently for the two sides.

2) It is able to allow tolerances of the welding gap of almost $\pm 1 \mathrm{~mm}$, while preserving a superior quality. It's worth notice that so far (20 magnets welded) SST passes have never failed.

3) Once a wire is properly qualified and the welding law (the set of parameters) defined in real conditions, the welding

${ }^{2}$ An Italian consortium (CTE Sistemi and CSA), that selected Lincoln for the welding equipment and Servorobot Canada for the gap reading and all automatic controls. itself is very reproducible and controllable allowing the pre-stress to be as uniform as requested.

4) The equipment for SST and MIG is the same, with an obvious advantage (MIG is a necessary choice for the filling passes to satisfy the time constraints).

Just to compare, TIG allows gap variation of few tenths of a $\mathrm{mm}$ and MIG is not able to guarantee the same level and uniformity of pre-stress obtained by SST.

The gap is read through a laser beam and all information is stored during a dry pass. All the welding operations will take, once sufficient experience is gained, some 8 hours, and 5-6 hours are necessary for the other press operations: magnet insertion, alignment, curvature measurements, etc. In total each company should be able to weld up to 4 to 5 magnets/week with a two-shift working day.

Finally, as part of the large tooling it is worth mentioning two, among others, measuring devices that are key elements in the success of the Project:

- The $15 \mathrm{~m}$ long presses to measure the coil size and their compressive elastic modulus. All procured by CERN, including the software for analysis and data viewer, they are an essential tool in keeping under control the geometry of the coils and then to respect both the very tight tolerances on the field quality and the optimal pre-stress window where we expect good quench performance.

- The Laser Tracker that is fundamental to measure the curvature, planarity, twist, and inter-aperture distance of the magnets, all along the $15 \mathrm{~m}$ long narrow Cold Bore Tube (CBT), with the requested $0.1 \mathrm{~mm}$ precision. It is also essential to measure the position (and actually to facilitate in the positioning during their assembly), of the all "3-D" components, i.e., at the magnet extremities. As previously said there are elements that need to be positioned and welded at $\pm 0.3 \mathrm{~mm}$ distance from the ideal line that is defined by the center of the CBT. Although difficult to achieve in an industrial environment that looks for quality but also time schedule, the Laser Tracker is proving to be essential, without which the required geometry and alignment of these magnets cannot be guaranteed.

\section{Quality Assurance (QA) and Production Control}

A superconducting magnet as complicated as the MB is a total quality product: any error will eventually result in a reduced performance or in complete failure, and it is therefore necessary to assure full traceability of all materials and their conformity to specifications.

1) MTF (Manufacturing and Test Folder): Developed at CERN [18], and called also Traveler (but it is much more than a Traveler) is a very useful tool that is based on the LHC QA general plan and the manufacturer QA plan. Through the MTF, CERN and manufacturers share:

- Traceability of all components, either supplied by CERN or by the companies themselves.

- Inspection and Test Plan.

— Workflow Diagram Structure.

- Assembly Breakdown Structure. 
- All measurement and test results.

- Handling of Non Conformities (ABS).

- Handling of all Technical Changes.

To describe the MTF in the detail would go beyond the scope of this paper. It suffices to underline that it contains a full description of a magnet, from the certification of the NbTi alloy employed for that particular lot of cables, to the measurement of the field quality of the magnet, passing through all electrical tests and all eventual deviations from the specification in terms of positioning of conductor, coil size, curvature of the magnets, etc. So the MTF is the complete description of a magnet and all its properties relevant for the construction, performance and its suitability for installation. To check that the MTF is properly compiled and other QA issues, since the beginning of 2002 we have resident inspectors in the three manufacturing companies and in the two main cabling facilities.

2) Relational Database: On the top of MTF, using its very important $\mathrm{ABS}$, we are building a relational database to allow correlating properties of the entire magnet production, or a particular subset, to component properties, process or tooling modifications. In this respect the relational database is the necessary tool for a complete analysis that we need not only to assess the magnet but also to drive construction and to allow to take corrective actions in a sufficient time. This is possible as many tolerances are statistical: we can accept a certain window for the average value and a certain standard deviation. We have to track trends, to assure that the extremities of the normal distribution are not filled too quickly. Thanks to this we can even accept out of tolerance magnets, if their number remains inside the target statistical limits.

\section{The Ultimate Production Steering Tool: Magnetic Field}

An example of the above is the introduction of magnetic field measurements at the companies at various stages: after coil collaring and after Cold Mass assembly. By means of suitable moles and dedicated equipment capable to measure fields of a few $\mathrm{mT}$, with a relative accuracy of better than $10^{-4}$, the harmonic content is measured together with the integrated transfer function: BL/I (Tm/A), an important parameter because all 154 dipoles in an arc are powered in series. Through these measurements done on collared coils, and thanks to the fact that there is a very good correlation between warm and cold measurements, we have been able to anticipate that the main harmonics were not quite right and to take corrective action, i.e., to modify the coil geometry by changing the copper wedges of the inner layer by few hundreds of $\mu \mathrm{m}$ [19]. In Fig. 5 a major harmonic, $b_{3}$, is plotted versus the number of collared coils and compared with the limit allowed by beam optics requirements [20]. Due to production time lag, we have at this moment more than 30 collared coils measured but we have only 10 magnets tested cold. Therefore the warm measurements at early stage are essential for a timely reaction.

Through field measurements on collared coils we have been able to assess as early as November 2001 that the magnetic length not only stays inside the required limits, but that it does not show any systematic difference among dipoles manufactured by different companies, as shown in Fig. 6. This has al-

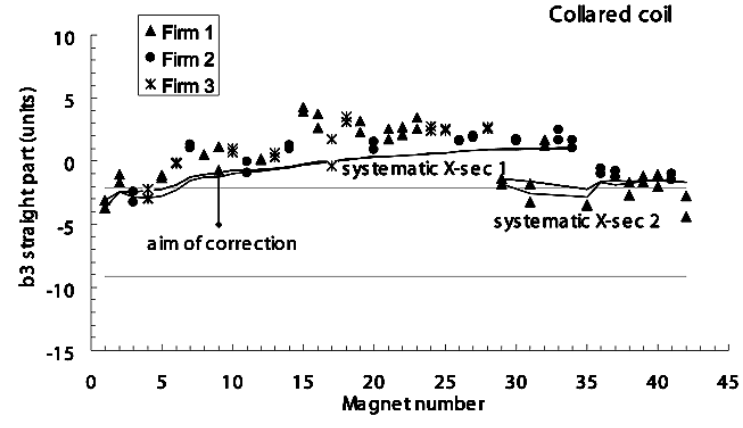

Fig. 5. Sextupole (main harmonic) of collared coils for the pre-series production. Note the effect of cross-section change and its delayed effect. Limits between -2 and -9 units ( 1 unit: $=10^{-4}$ of the main field).

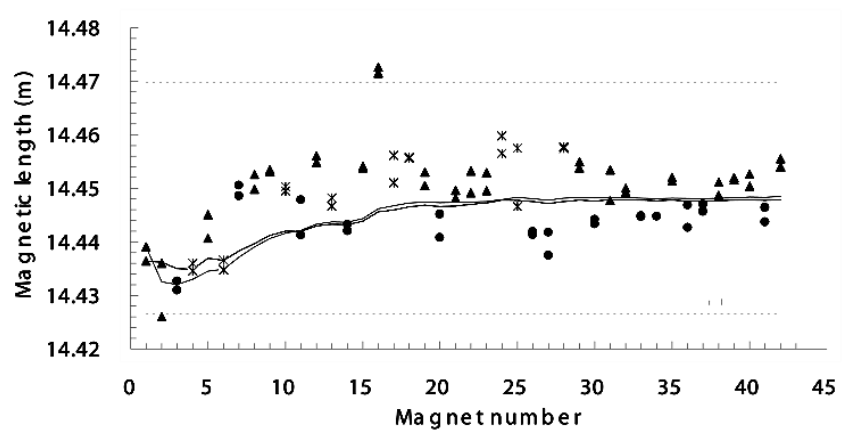

Fig. 6. Magnetic length of collared coils, showing no systematic effect. Limits are 14.425 and $14.47 \mathrm{~m}$.

lowed CERN to abandon the constraints of requiring magnets of same manufacturer for a given octant, making easier the negotiation of the main tender and greatly improving efficiency of tunnel installation.

Field measurements at the companies not only are useful to control the field quality. They have been very useful to detect:

- An important assembly error in one company at a very early stage, allowing to take immediate corrective actions (we have been able to indicate the position and the source of the fault).

- Coil waviness, beyond the target limit of $30 \mu \mathrm{m}$, of one company that was traced back to differences in creep behavior between the polyimide used for cable insulation of the prototypes and the first two pre-series magnets and the polyimide used later. The difference in creep could not be easily accommodated by the curing mold used in that company. As a consequence, the company agreed to modify the curing mould and now the situation has improved.

Finally, it is important to remark that as a final check before shipping, we have in the companies a measurement of the distance between: A) the CBT axis, that is the mechanical axis used as reference for geometric reference, fiducials, marking interconnection and alignment in the tunnel; B) the coil symmetry axis. This last is defined as the zero field line when the coils are energized in the so-called Quadrupole Configured Dipole mode [21]. This distance is very important since it can affect the aperture of the machine and the luminosity depends strongly on preserving both mechanical and dynamic apertures. 


\section{PRE-SERIES Dipoles Results}

Results on 13 pre-series dipole magnets already delivered to CERN, cryostated and then cold tested, can be summarized as follows:

- Ten magnets reached $9 \mathrm{~T}$, the ultimate field, some of them with very little training. Also the retraining properties were very good. Some of them, after thermal cycle went straight to $9 \mathrm{~T}$ with no quench.

- The field performance when cold is generally good, as anticipated by warm measurements, [19], although some harmonics need to be pulled inside the limits and a more solid correlation between properties measured on cable short samples and magnet properties (namely: magnetization, snap-back and interstrand resistance) needs to be established before the main series starts. Through this, in principle we can fully foresee the properties of a magnet and steer the production to make sure that all magnets are almost interchangeable, avoiding sorting, a major goal to make the installation in the tunnel easier and faster.

- One magnet (N. 2002) was not powered because of problems in the quench heaters.

- One magnet (N. 1005) was limited to a performance of about $6 \mathrm{~T}$, without any training or indication of mechanical defect, due to a local fault. The magnet was disassembled, the suspected coil de-wound and a region with at least 30 cold welds in the superconducting cable (out of 36 strands) was found. This cable was manufactured in July 2000 (the magnet was tested in May 2002) at the very beginning of the cable series production and, evidently, it escaped all controls (at that time not so tight as they are now: for example we did not have resident inspectors). Investigations are under way and an audit to review the identification and archiving system of cold welds has been called for the next month.

- One magnet (N. 2001) was limited just beyond the nominal field, it never reached the ultimate field, and showed a strange behavior: it appeared limited by a mechanical defect at $1.9 \mathrm{~K}$ and limited by heat dissipation effects at $4.2 \mathrm{~K}$. We suspect that a splice may be slightly defective (see footnote n.1).

The above mentioned results, see also Fig. 7, enable us to affirm that the design is sound for the nominal field operation, $8.33 \mathrm{~T}$ and that there is room to go beyond the nominal. Considering that we and the companies are still in learning phase it is reasonable to expect that the vast majority of the magnets can work at $9 \mathrm{~T}$, although operating at such very high level will actually depend on a number of issues like: a) effective radiation heating inside the tunnel; $b$ ) number of training quench allowed in the tunnel; c) trade off among energy level and integrated luminosity (i.e., beam delivery time).

All together we can affirm that, although there is certainly room for improvement, especially in the area of control and QA, all the three companies can to start series production of the main dipoles at the beginning of 2003 .

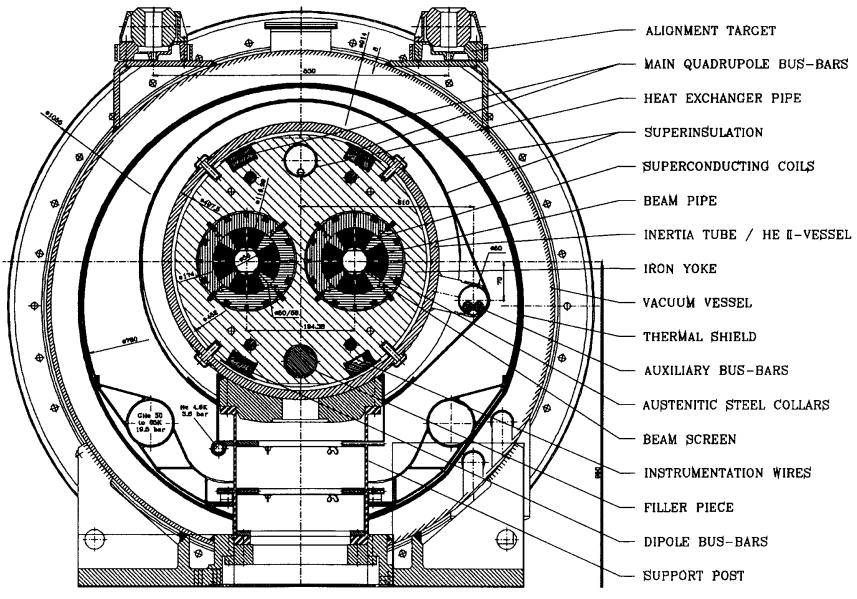

Fig. 7. Results obtained in the first training cycle (at $1.9 \mathrm{~K}$ ) for the pre-series dipole tested so far.

\section{Production Status, Planning And Conclusion}

The LHC Project relies heavily on the delivery schedule of the main dipoles and quadrupoles, covering $40 \%$ of the whole LHC cost, determining its performance and filling $70 \%$ of the tunnel: somehow they give the clock to the entire Project. In this respect keeping the schedule is almost as essential as keeping the quality. The technology transfer to industry is almost completed, with minor improvements still needed on the cold mass assembly, which has been transferred to Industry in the last six months. Improvement in the use of Laser tracker and the demonstration that the welding press can reach the established goal in terms of productivity are the major challenge in the second part of 2002.

Fine tuning of all procedures, especially of shimming and coil sizing, uniformity among companies and improvement of electrical tests for quench heaters (an area in which we had a lot of nonconformities in the past) are under way.

From the point of view of the components the area of higher risk is certainly superconducting cables. The production of cable is suffering a year of delay, like the cold mass construction, and a number of actions have been taken to improve the situation

- Reallocation of production among companies.

- Ordering (from CERN directly) of a spare cabling machine, to be installed in January 2003.

- Revision of some contractual terms to ease production.

- Establish strong monitoring to anticipate problems.

- Inspection in the companies that allowed identifying the main source of problems as being contamination from spurious small particles.

These actions are permitting the LHC to pass through the last crises (a major breakdown of one of the cabling machines) without endangering the magnet production and, eventually, will allow constituting a six month buffer stock of cables.

The delivery of cables as anticipated today should have always six months lead over the "just-on-time," i.e., the last cable is scheduled to be shipped by summer 2005. In Fig. 8 a realistic schedule of dipoles, as recently re-discussed with the companies, is plotted. At present we are delayed and we anticipate that by end of the year we'll be missing some 25 dipoles with respect to the planning of beginning 2002. However, the production rate at full blast will be some 8 dipoles per week, i.e., the delay of 


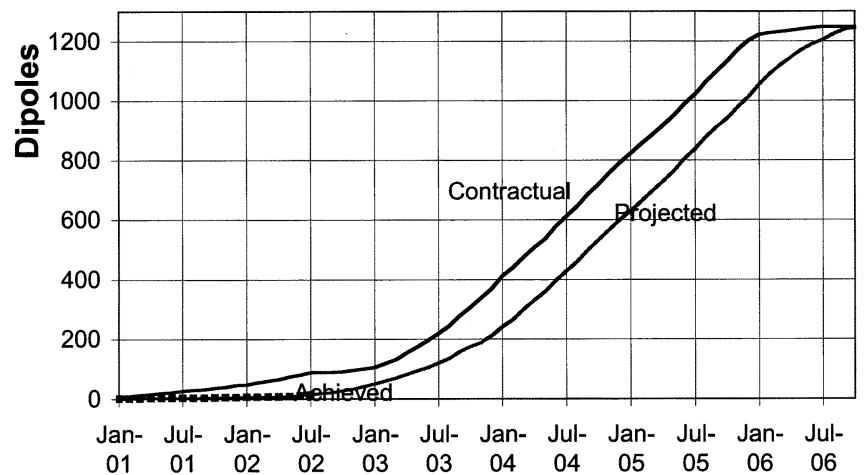

Fig. 8. Delivery of Main Dipoles. Today 13 magnets have been delivered.

this year can be recovered in 3 weeks work. A serious assessment of the reliability of the schedule can be done eventually in spring 2003, when all the tooling, in particular the winding lines, will be installed and working at maximum capacity.

As concerns the MQ, their production is also suffering about one year delay with respect to the original delivery plan. However the first industrially manufactured MQ has been delivered to CERN at the end of July and the second is foreseen by August 2002. If their performance will be as expected then the real production can start, according to a new schedule that foresees the last quadrupole delivered to CERN by 2005 .

In conclusion, the Project of the LHC Main Magnets is suffering of about one year delay with respect to the original plan of 2000 (closing of LEP). One year delay is also appearing in some of the major components, like the superconducting cables (but it is not so for other components such as yoke laminations, generating storage problems). However, now that the large scale production of cables has started and the magnets are exploiting successfully the pre-series phase, we are ready to enter into the series phase at the beginning of the 2003. Magnet design and construction procedures are adequate for the nominal field and operation at ultimate field may be possible. The present realistic plan foresees that the magnet delivery is finished before end of 2006, allowing beam acceleration in 2007.

\section{ACKNOWLEDGMENT}

The author is indebted to many colleagues of the CERN LHC division and in particular to: M. Bajko, P. Fessia, C. Lanza, P. Lienard, A. Siemko, E. Todesco, T. Tortschanoff and the superconducting cable team. Thanks are due to the companies for their kind collaborations.

\section{REFERENCES}

[1] P. Lefrevre and T. Pettersson, Eds., "The large hadron collider-conceptual design,", report CERN/AC/95-05 (LHC), October 20, 1995.
[2] L. R. Evans, "The large hadron collider-Present status and presents," IEEE Trans. on Applied Superconductivity, vol. 10, no. 1, pp. 44-48, March 2000.

[3] R. Scmidt, "Status of the LHC," in EPAC02 (European Particle Accelerator Conference, submitted for publication in the Proceedings, submitted for publication.

[4] D. Hagedorn, D. Leroy, and R. Perin, "Toward the development of high field superconducting magnets for a hadron collider in the LEP tunnel," in Proc. of the 9th Int. Conference on Magnet Technology (MT-9), Zurich, C.H. Villigen: SIN publisher, CH-5234, September 9-13, 1985, pp. 86-91.

[5] L. Rossi, "State-of-the-art accelerator magnets," IEEE Trans. On Applied Superconductivity, vol. 12, no. 1, pp. 219-227, March 2002.

[6] _ - "Report on the magnet-cryogenic-vacuum working group: Possible options and cost for superconducting magnets and cryogenics," in Hadron Colliders at the Highest Energy and Luminosity, A. G. Ruggiero, Ed: World Scientific, pp. 133-140.

[7] I. J. Billan, M. Bona, L. Bottura, D. Leroy, O. Pagano, R. Perin, D. Perini, F. Savary, A. Siemko, P. Sievers, G. Spigo, J. Vlogaert, L. Walckiers, C. Wyss, and L. Rossi, "Test results on long models and full scale prototype of the second generation LHC arc dipoles," IEEE Trans. on Applied Superconductivity, vol. 9, no. 2, pp. 1039-1044, June 1999.

[8] R. Perin, "First results of the high field magnet development for the large hadroncollider," in Proc. of the 11th Int. Conf. on Magnet Technology (MT-11). Tsukuba, Japan: Elsevier Publisher, 1989, pp. 42-47.

[9] S. Ramberger and S. Russenschuck, "Genetic algorithms for the optimal design of superconducting accelerator magnets," in Proc. of EPAC98, LHC project report 275.

[10] C. Lanza and D. Perini, "Characteristics of the austenitic steels used in the LHC main dipoles,", CERN LHC Project Report 537, March 6, 2002.

[11] P. Fessia, D. Perini, S. Russenschuck, C. Voellinger, R. Vuillermet, and C. Wyss, "Selection of the cross-section design for the LHC main dipole," IEEE Trans. on Applied Superconductivity, vol. 10, no. 1, pp. 65-68, March 2000.

[12] M. Baijko, P. Fessia, and D. Perini, "Statistical studies of the robustness of the LHC main dipole mechanical structure," IEEE Trans. on Applied Superconductivity, vol. 10, no. 1, pp. 77-80, March 2000.

[13] S. Redaelli, "Analysis of the magnetic field perturbations in dipoles and quadrupoles of the large hadron colliders (LHC)," Thesis, Physics Dept. of the University of Milan, via Celoria 16, 20133 Milano-I.

[14] J. D. Adam et al., "Status of the LHC superconducting cable mass production," IEEE Trans. on Applied Superconductivity, vol. 12, no. 1, pp. 1056-1062, March 2002.

[15] P. Fessia, D. Perini, R. Vuillermet, and C. Wyss, "Comparative study of different designs of the mechanical structure of the LHC main dipole,", CERN LHC Project Note 288, April 18, 2002.

[16] T. Tortschanoff et al., "The short straight sections for the LHC," in Proc. PAC97 (Particle Accelerator Conference), Vancouver, B.C., Canada, 1997.

[17] J. Billan, V. Remondino, A. Siemko, N. Smirnov, T. Tortschanoff, M. Peyrot, J. M. Rifflet, and F. Simon, "Performance of prototypes and start up of series fabrication of the LHC arc quadrupoles," in Proc. PAC 2001 (Particle Accelerator Conference), Chicago, June 2001.

[18] C. Delamare, A. Jimeno, S. Mallon Amerigo, E. Manola-Poggioli, P. Martel, B. Rousseau, and D. Widegren, "Manufacturing and test folder: MTF," in EPAC02, Paris, June 2002, submitted for publication in the Proceedings.

[19] L. Bottura, S. Pauletta, V. Remondino, S. Sanfilippo, W. Scandale, and E. Todesco, "Status report on field quality in the main LHC dipoles,", Geneva, LHC-Project-Report-579, CERN, July 2, 2002. submitted for publication in the Proc. of EPAC02, Paris, June 2002.

[20] W. Scandale and E. Todesco, private communication CERN,-LHC.

[21] J. Billan, F. Fischer, P. Galbraith, J. Garcia Perez, N. Mermillod, and G. Patti, "An AC field static system for measuring the magnetic axis of LHC superconducting magnets in warm conditions," CERN, LHC-MMS internal note N. 2000-1, January 2000 